\title{
Influence of temperature fluctuations on the shape of the spectral continuum
}

\author{
N. A. Silant'ev ${ }^{1}$ and G. A. Alexeeva ${ }^{2}$ \\ ${ }^{1}$ Instituto Nacional de Astrofísica, Óptica y Electrónica, Apartado Postal 51 y 216, CP 7200, Puebla, Pue., México \\ e-mail: silant@inaoep.mx \\ 2 Main Astronomical observatory of Russian Academy of Sciences, Pulkovskoe shosse 65, S.-Petersburg 196140, Russia
}

Received 9 April 2007 / Accepted 1 November 2007

\section{ABSTRACT}

\begin{abstract}
We investigate the influence of stochastic temperature fluctuations on the observed spectra in continuum. Temperature fluctuations exist in the photospheres and atmospheres of stars, in interstellar media, and in the vicinity of AGN and quasars. In these cases we observe the mean values of intensities from the objects. The particular calculations were made for the frequently used model of semi-infinite turbulent homogeneous atmosphere with the Planck source function. We show that temperature fluctuations $T^{\prime}$ change the spectral distribution of observed radiation in all regions of spectra, especially in the ultraviolet region, where they increase the mean intensity compared with the Planck intensity $B_{v}\left(T^{(0)}\right)$ at the mean temperature $T^{(0)}$. In the maximum of Planck's spectrum, the difference between the Planck intensity $B_{v}\left(T^{(0)}\right)$ and the observed mean intensity $I_{v}^{(0)}$ can also be large (about 5-30\%). This difference can be negative or positive depending on the particular form of the absorption coefficient. Our results demonstrate that the turbulencerelated small-scale temperature fluctuations contribute to the overall deviations from the Planck intensity. The temperature fluctuations change the observed $U-B, B-V$, etc. colour indices. Our simple technique can also be used to analyze of the radiation spectra from all sources, not only for the thermal Planck radiation.
\end{abstract}

Key words. radiative transfer - turbulence - line: formation - line: profiles

\section{Introduction}

Many photospheres and atmospheres of stars have the chaotic (turbulent) motions of the gas (see, for example, Gray 1992; Sobolev 1969). The motion of interstellar matter is also frequently stochastic, especially in the vicinity of various active sources such as AGN, supernova explosions, and quasars. The stochastic motions of the matter are followed by corresponding temperature fluctuations (see Tatarskii 1967).

In turbulent (more generally, stochastic) media, the absorption coefficient $\alpha_{v}(T)$, the intensity of radiation $I_{v}$, the temperature $T$, and the number density $N$ of scattering particles are also the stochastic values. They are characterized by its mean values $-\alpha_{v}^{(0)}, I_{v}^{(0)}, T^{(0)}, N^{(0)}$, and by the fluctuating components $\alpha_{v}^{\prime}, I_{v}^{\prime}, T^{\prime}$, and $N^{\prime}$. So, we have $\alpha_{v}=\alpha_{v}^{(0)}+\alpha_{v}^{\prime}, I_{v}=I_{v}^{(0)}+I_{v}^{\prime}, T=$ $T^{(0)}+T^{\prime}$, etc., where the mean values of fluctuating quantities are equal to zero; for example, $\left\langle T^{\prime}\right\rangle=0$.

The visual semisphere of a star contains many turbulent (stochastic) cells; i.e. we really only observe the mean radiative flux. The only exception is the Sun. More detailed discussion of this subject is given by Levshakov \& Kegel (1997). The temperature fluctuations were observed directly in the photosphere of the Sun (see Stix 1992). Observed local variations in the surface radiation intensity allow us to estimate the corresponding fluctuations in the temperature. It turns out that the solar temperature fluctuations $T^{\prime}$ are very small compared to the mean temperature $T^{(0)}: T^{\prime} / T^{(0)} \simeq 0.03$. We demonstrate that even such low values for temperature fluctuations give rise to rather large changes in the observed integral spectra of radiation. An especially large effect exists for the ultraviolet range of wavelengths.
This can be demonstrated in a simple model of two realizations. Let us observe the Planck intensity (in Wien's region of wavelengths) in the first realization with the temperature $T^{(0)}+T^{\prime}$ and in the second realization with the temperature $T^{(0)}-T^{\prime}$.

The mean value of the intensity is

$$
\begin{aligned}
\left\langle I_{v}\right\rangle \equiv I_{v}^{(0)}= & \frac{1}{2}\left(\frac{2 h v^{3}}{c^{2}}\right)\left[\exp \left(-\frac{h v}{k\left(T^{(0)}+T^{\prime}\right)}\right)\right. \\
& \left.+\exp \left(-\frac{h v}{k\left(T^{(0)}-T^{\prime}\right)}\right)\right] \\
\cong & I_{v}\left(T^{(0)}\right) \cosh \left(\frac{h v}{k T^{(0)}} \cdot \frac{T^{\prime}}{T^{(0)}}\right) \geq I_{v}\left(T^{(0)}\right) .
\end{aligned}
$$

Here and in what follows, we suppose inequality $T^{\prime} \ll T^{(0)}$. It is seen from Eq. (1) that the greater the parameter $h v / k T^{(0)}$, the larger the difference between $I_{v}^{(0)}$ and $I_{v}\left(T^{(0)}\right)$; hence for $h v / k T^{(0)}=10$ and $T^{\prime} / T^{(0)}=0.05$, we have the $13 \%$ increase, and the increase is much larger for $h v / k T^{(0)}=20$, about $54 \%$. This is purely statistical effect - the mean value of observed fluctuating intensities is higher than the intensity corresponding to the mean temperature. Because we only observe the mean value of the radiation flux, this statistical effect has to be taken into account.

The deviation in the mean intensity $I_{v}^{(0)}$ from the Planck intensity $B_{v}\left(T^{(0)}\right)$ due to the temperature fluctuations occurs in all parts of a spectrum, including the maximum of the spectrum and the far-infrared wavelengths where both the increase and the decrease of the intensity can be observed.

The goal of this paper is to give simple analytical consideration of the various situations where small temperature 
fluctuations give rise to fairly large changes in the spectra. The authors plan to apply the theory to some observing results in subsequent papers. Below we give the detailed consideration of these statistical effects.

\section{Radiative transfer equation for the mean intensity}

Silant'ev (2005) has shown that the mean intensity $I_{v}^{(0)}(\boldsymbol{r}, \boldsymbol{n})$ satisfies the usual radiative transfer equation with the effective kinetic coefficients and the averaged source function:

$$
\begin{aligned}
(\boldsymbol{n} \nabla) I_{v}^{(0)}(\boldsymbol{r}, \boldsymbol{n})= & -\alpha_{v}^{(\mathrm{eff})} I_{v}^{(0)}(\boldsymbol{r}, \boldsymbol{n}) \\
& +\beta_{v}^{(\mathrm{eff})} \int \mathrm{d} \boldsymbol{n}^{\prime} \Phi\left(\boldsymbol{n} \boldsymbol{n}^{\prime}\right) I_{v}^{(0)}\left(\boldsymbol{r}, \boldsymbol{n}^{\prime}\right)+\left\langle\alpha_{v}^{(a)} B_{v}(T)\right\rangle,
\end{aligned}
$$

where $B_{v}(T)$ is the Planck function, $\alpha_{v}^{(a)}$ the true absorption coefficient, and $\Phi\left(\boldsymbol{n} \boldsymbol{n}^{\prime}\right)$ the scattering phase function (for isotropic scattering this is simply $1 / 4 \pi$ ). The total extinction factor $\alpha_{v}=$ $N \sigma_{v}^{(s)}+\alpha_{v}^{(a)}$, where $\sigma_{v}^{(s)}$ is the scattering cross-section and $N$ the number density of the scattering particles. The effective kinetic coefficients $\alpha_{v}^{(\mathrm{eff})}$ and $\beta_{v}^{(\mathrm{eff})}$ have the following form:

$\alpha_{v}^{(\mathrm{eff})}=\alpha_{v}^{(0)}\left(1-\frac{\left\langle\left(\alpha^{\prime}\right)^{2}\right\rangle}{\left(\alpha_{v}^{(0)}\right)^{2}} \tau_{1} t_{\alpha}\right)$,

$\beta_{v}^{(\mathrm{eff})}=N^{(0)} \sigma_{v}^{(s)}\left(1-\frac{\left\langle\alpha^{\prime} N^{\prime}\right\rangle}{\alpha_{v}^{(0)} N^{(0)}} \tau_{1} t_{\alpha N}\right)$.

Here $\tau_{1}=\alpha_{v}^{(0)} R_{1}$ is the mean total optical depth of the turbulent correlation length $R_{1}$. Remember that the characteristic scale $R_{1}$ is determined from the condition that, outside the region $\sim R_{1}$, the fluctuating quantities are practically uncorrelated one with the other (see Tatarskii 1967). The coefficients $t_{\alpha} \simeq 1$ and $t_{\alpha N} \simeq 1$ are determined by the particular forms of turbulent correlation functions, for example:

$t_{\alpha N}=\int_{0}^{\infty} \mathrm{d} x A_{\alpha N}(x)$,

$\left\langle\alpha^{\prime}(s) N^{\prime}\left(s^{\prime}\right)\right\rangle=\left\langle\alpha^{\prime}(s) N^{\prime}(s)\right\rangle A_{\alpha N}\left(R / R_{1}\right)$.

The correlation function $A_{\alpha N}\left(R / R_{1}\right)$ depends on the difference $R=\left|s-s^{\prime}\right|$ of distances along the line of sight $\boldsymbol{n}$ and is equal to unity at $R=0$. The value $\left\langle\alpha^{\prime}(s) N^{\prime}(s)\right\rangle$ is the corresponding mean value at the s-point. Note that one frequently uses the model $A_{\alpha}=\exp \left(-R / R_{1}\right)$, which gives $t_{\alpha}=1$.

The transfer Eq. (2) is valid if $\tau_{1} \ll 1$. This case evidently corresponds to the small-scale turbulence in the atmosphere. If this condition is not valid, then the propagation of radiation is described by the effective optical depth $\tau_{v}^{\text {(eff) }}$ (see Silant'ev 2005; and Silant'ev et al. 2006), which depends nonlinearly on the distances.

In the theory of stellar photospheres (see Gray 1992) the scattering term in Eq. (2) can usually be omitted. We also neglect this term and consider only the source term $\left\langle\alpha_{v}^{(a)} B_{v}(T)\right\rangle$ in deatail. Of course, the scattering term cannot be omitted if the process of the Thomson scattering and the atomic and molecular scatterings in stellar atmosphere play important roles (see Mihalas 1978). Furthermore, for brevity, we denote the $\alpha_{v}^{(a)}$-coefficient simply as $\alpha_{v}$.

Strictly speaking, the usual hydrostatic theory of the stellar photospheres should be based on the radiative transfer Eq. (2), which takes the temperature fluctuations into account.
We shall see that one holds the inequality $\left\langle\left(\alpha^{\prime}\right)^{2}\right\rangle /\left(\alpha_{v}^{(0)}\right)^{2} \ll 1$ for small temperature fluctuations, and the coefficient $\alpha_{v}^{\text {(eff) }}$ practically coincides with the mean absorption coefficient $\alpha_{v}^{(0)}$. The latter depends on the mean temperature $T^{(0)}$ and the level of the temperature fluctuations

$\eta=\frac{\sqrt{\left\langle\left(T^{\prime}\right)^{2}\right\rangle}}{T^{(0)}}$

and does not coincide with the value $\alpha_{v}\left(T^{(0)}\right)$, i.e. $\alpha_{v}^{(0)}=$ $\alpha_{v}^{(0)}\left(T^{(0)}, \eta\right)$. Note that in general case both $T^{(0)}$ and parameter $\eta$ depend on the position $\boldsymbol{r}$ in an atmosphere.

Frequently (see Rybicki \& Lightman 1979), one uses the dimensionless optical depth $\mathrm{d} \tau_{v}=\alpha_{v}^{(0)} \mathrm{d} s$ and denotes the last source term in Eq. (2) as $S_{v}$. In this notion the source term in Eq. (2) acquires the form

$S_{v}\left(T^{(0)}, \eta\right)=\frac{\left\langle\alpha_{v}\left(T^{(0)}+T^{\prime}\right) B_{v}\left(T^{(0)}+T^{\prime}\right)\right\rangle}{\alpha_{v}^{(0)}\left(T^{(0)}, \eta\right)}$,

which is different from the usual term $B_{v}\left(T^{(0)}\right)$ in the standard stellar photosphere theory.

Thus, the new transfer equation due to coefficients $\alpha_{v}^{(0)}$ and $S_{v}$ depends on the new physical parameter $\eta-$ the level of temperature fluctuations. Of course, all the known methods of the usual hydrostatic photosphere theory can be used to construct the models of photospheres that take the existence of the temperature fluctuations into account. In particular, such a modification of the photosphere theory may be useful for improving the coincidence with the observed ultraviolet excesses (as in the Sun).

For the crude estimates of the influence of the temperature fluctuations on the spectra of cosmic objects, we use the simple model of a homogeneous turbulent semi-infinite medium with given level of the temperature fluctuations. In this case the source function $S_{v}\left(T^{(0)}, \eta\right)$ does not depend on the optical depth, and the emerging radiation intensity is equal to this function (see Rybicki \& Lightman 1979). To estimate the difference between the $S_{v}$ - function and the Planck function $B_{v}\left(T^{(0)}\right)$, we present expression (7) in the form

$S_{v}\left(T^{(0)}, \eta\right) \equiv f\left(T^{(0)}, \eta\right) B_{v}\left(T^{(0)}\right)$,

where the dimensionless distortion function $f_{v}\left(T^{(0)}, \eta\right)$ characterizes the degree of the difference from the Planck spectral distribution:

$f_{v}\left(T^{(0)}, \eta\right)=\frac{\left\langle\alpha_{v}\left(T^{(0)}+T^{\prime}\right) B_{v}\left(T^{(0)}+T^{\prime}\right)\right\rangle}{\alpha_{v}^{(0)}\left(T^{(0)}, \eta\right) B_{v}\left(T^{(0)}\right)}$

It is clear that this function does not depend on the temperatureindependent factors in $\alpha_{v}(T)$ and $B_{v}(T)$.

There are many expressions for the absorption coefficients that describe the absorption of radiation in various physical situations. As typical examples, we consider below only two forms of $\alpha_{v}(T)$, those that are simplest for calculations. The first absorption coefficient is

$\alpha_{v}(T)=C_{v} T \exp \left(-\frac{I}{k T}\right)\left[1-\exp \left(-\frac{h v}{k T}\right)\right]$.

This coefficient is used in stellar photosphere theory (see Gray 1992) for describing free-free transitions in the hydrogen photospheres $(I=13.6 \mathrm{eV}$ is the ionization energy of the hydrogen atom). 
We introduce here the dimensionless parameters:

$a=\frac{k T^{(0)}}{h v} \simeq \frac{\lambda(\mu \mathrm{m}) T^{(0)}(\mathrm{K})}{14388} \simeq 0.0695 \lambda(\mu \mathrm{m})\left(\frac{T^{(0)}}{10^{3}}\right)$,

$b=\frac{k T^{(0)}}{I} \simeq 0.00634\left(\frac{T^{(0)}}{10^{3}}\right)$.

The second absorption coefficient

$\alpha_{v}(T)=D_{v} \frac{1}{\sqrt{T}}\left[1-\exp \left(-\frac{h v}{k T}\right)\right]$

also describes the free-free transitions in the electron-ion plasma (see Rybicki \& Lightman 1979), where the concentrations of free electrons and ions are considered as given values (the $D_{v}$-term is proportional to them). The factor $(1-\exp (-h v / k T))$ is a known correction Einstein's factor for taking the stimulated emission into account (see Rybicki \& Lightman 1979). In Wien's range of the wavelengths, it tends to unity, but in the Rayleigh-Jeans region it gives the $T^{-3 / 2}$ - dependence of the absorption coefficient. The coefficients (10) and (13) are used in astrophysics in various regions of frequency $v$ and temperature $T$ (see, for example Gray 1992; and Rybicki \& Lightman 1979).

In the next section we calculate the distortion function $f_{v}\left(T^{(0)}, \eta\right)$ for these absorption coefficients.

\section{The results of calculations}

We assume that the probability that small temperature fluctuations have a Gaussian form (see Van Kampen 1981). In this case the mean value of a function $G(T)$ can be calculated from the formula:

$$
\begin{aligned}
\langle G(T)\rangle & =\frac{1}{\sqrt{2 \pi\left\langle T^{\prime 2}\right\rangle}} \int_{0}^{\infty} \mathrm{d} T G(T) \exp \left(-\frac{\left(T-T^{(0)}\right)^{2}}{2\left\langle T^{\prime 2}\right\rangle}\right) \\
& =\frac{1}{\sqrt{2 \pi} \eta} \int_{-1}^{\infty} \mathrm{d} x G\left(T^{(0)}(1+x)\right) \exp \left(-\frac{x^{2}}{2 \eta^{2}}\right) \\
& \cong \frac{1}{\sqrt{2 \pi} \eta} \int_{-\infty}^{\infty} \mathrm{d} x G\left(T^{(0)}(1+x)\right) \exp \left(-\frac{x^{2}}{2 \eta^{2}}\right)
\end{aligned}
$$

Here $x=\left(T-T^{(0)}\right) / T^{(0)}$. We use for the calculations the last term in Eq. (14), which is available for low values of the parameter $\eta$. Practically, this is possible for $\eta \leq 0.2$.

According to Eq. (14), we calculate the values $\alpha_{v}^{(0)}$ and $\left\langle\alpha_{v}(T) B_{v}(T)\right\rangle$ using the following explicit formulae:

$$
\begin{aligned}
& \left\langle\alpha_{v}(T)\right\rangle=\frac{1}{\sqrt{2 \pi} \eta} \int_{-\infty}^{\infty} \mathrm{d} x \alpha_{v}\left(T^{(0)}(1+x)\right) \exp \left(-\frac{x^{2}}{2 \eta^{2}}\right) \\
& \left\langle\alpha_{v}(T) B_{v}(T)\right\rangle=\frac{2 h v^{3}}{c^{2}} \frac{1}{\sqrt{2 \pi} \eta} \int_{-\infty}^{\infty} \mathrm{d} x \alpha_{v}\left(T^{(0)}(1+x)\right) . \\
& \exp \left(-\frac{x^{2}}{2 \eta^{2}}\right)\left[\exp \left(\frac{g}{1+x}\right)-1\right]^{-1} .
\end{aligned}
$$

The Wien approximation of Planck's function corresponds to $a \ll 1$. Remember that the maximum of the Planck function occurs at $\lambda(\mu \mathrm{m}) T^{(0)}(\mathrm{K}) \cong 2900$, i.e. corresponds to the value $a \cong 0.2$.

In the standard hydrostatic theory of stellar photospheres, one frequently uses the frequency averaged absorption coefficient $\overline{\alpha(T)} \propto T^{\gamma}$. In the ionized plasma for the Rayleigh-Jeans region of wavelengths, the absorption coefficient (13) $\alpha_{v} \propto T^{\gamma}$ with $\gamma=-3 / 2$. We give here the explicit formulae for the mean values of these type absorption coefficients:

$$
\begin{aligned}
\frac{\alpha_{v}^{(0)}}{\alpha_{v}\left(T^{(0)}\right)}= & 1+\frac{1}{2} \gamma(\gamma-1) \eta^{2} \\
& +\frac{1}{8} \gamma(\gamma-1)(\gamma-2)(\gamma-3) \eta^{4}+\ldots
\end{aligned}
$$

To calculate the value $\left\langle\left(\alpha_{v}^{\prime}\right)^{2}\right\rangle=\left\langle\alpha_{v}^{2}\right\rangle-\left\langle\alpha_{v}\right\rangle^{2}$, we need to know $\left\langle\alpha_{v}^{2}\right\rangle$ :

$$
\begin{aligned}
\frac{\left\langle\alpha_{v}^{2}\right\rangle}{\left(\alpha_{v}\left(T^{(0)}\right)\right)^{2}}= & 1+\gamma(2 \gamma-1) \eta^{2} \\
& +\frac{1}{2} \gamma(\gamma-1)(2 \gamma-1)(2 \gamma-3) \eta^{4}+\ldots
\end{aligned}
$$

These expressions give rise to the following result:

$$
\frac{\left\langle\left(\alpha_{v}^{\prime}\right)^{2}\right\rangle}{\left(\left\langle\alpha_{v}\right\rangle\right)^{2}} \cong \frac{\gamma^{2} \eta^{2}\left(1+\frac{1}{2}(\gamma-1)(3 \gamma-5) \eta^{2}\right)}{1+\gamma(\gamma-1) \eta^{2}+\frac{1}{2} \gamma(\gamma-1)\left(\gamma^{2}-3 \gamma+3\right)}
$$

This formula depends on $\eta^{2} \ll 1$. It means that the term inside the brackets in Eq. (3) is small and can be omitted, so we can take $\alpha_{v}^{(\text {eff })} \cong \alpha_{v}^{(0)}$. The numerical calculations for the absorption coefficients (10) and (13) confirm this result. Of course, we do not guarantee that this result exists for any forms of the absorption coefficients.

Using Wien's approximation of the Planck intensity $B_{v}(T) \propto$ $\exp (-h v / k T)$ and the explicit form of the absorption coefficient

$\alpha_{v}(T)=D_{v} T^{\gamma}\left[1-\exp \left(-\frac{h v}{k T}\right)\right]$

one can derive the analytical formula

$f_{v}\left(T^{(0)}, \eta\right)=\mathrm{e}^{g} \frac{\Phi_{0}(g, \eta) \Phi_{1}(g, \eta)-\Phi_{0}(2 g, \eta) \Phi_{1}(2 g, \eta)}{\Phi_{0}(0, \eta) \Phi_{1}(0, \eta)-\Phi_{0}(g, \eta) \Phi_{1}(g, \eta)}$,

where functions $\Phi_{0}(g, \eta)$ and $\Phi_{1}(g, \eta)$ have the forms:

$\Phi_{0}(g, \eta)=\frac{1}{\sqrt{1+2 g \eta^{2}}} \exp \left(\frac{g^{2} \eta^{2}}{2\left(1+2 g \eta^{2}\right)}\right)$

$$
\begin{aligned}
\Phi_{1}(g, \eta)= & {\left[1+\gamma \frac{g \eta^{2}}{1+2 g \eta^{2}}\right.} \\
& \left.+\frac{1}{2} \gamma(\gamma-1) \frac{\eta^{2}}{1+2 g \eta^{2}}\left(1+\frac{g^{2} \eta^{2}}{1+2 g \eta^{2}}\right)\right] \mathrm{e}^{-g}
\end{aligned}
$$

For brevity we use here the notion $g=1 / a$. It is seen that function $f_{v}\left(T^{(0)}, \eta\right) \equiv f_{v}(a, \eta)$ depends on the product $\lambda T^{(0)}$.

The analytical expression for $\left\langle\alpha_{v}(T)\right\rangle$ takes the form:

$\frac{\alpha_{v}^{(0)}\left(T^{(0)}, \eta\right)}{\alpha_{v}\left(T^{(0)}\right)}=\Phi_{0}(0, \eta) \Phi_{1}(0, \eta)-\Phi_{0}(g, \eta) \Phi_{1}(g, \eta)$.

If the expression (20) for $\alpha_{v}(T)$ contains the additional factor $\exp (-1 / b)$, i.e. looks like Eq. (10), then Eqs. (21) and (24) also take place with the evident substitutions: $g \rightarrow g+1 / b$ and $2 g \rightarrow 2 g+1 / b$. The approximate analytical formulae (21) and (24) represent $f_{v}$ and $\left\langle\alpha_{v}(T)\right\rangle$ rather well for small $\eta$ (up to $\eta=0.1)$ and can be used for the quick estimates in the range $0.03 \leq a \leq 0.2$. 
$\mathrm{f}(\mathrm{a})$

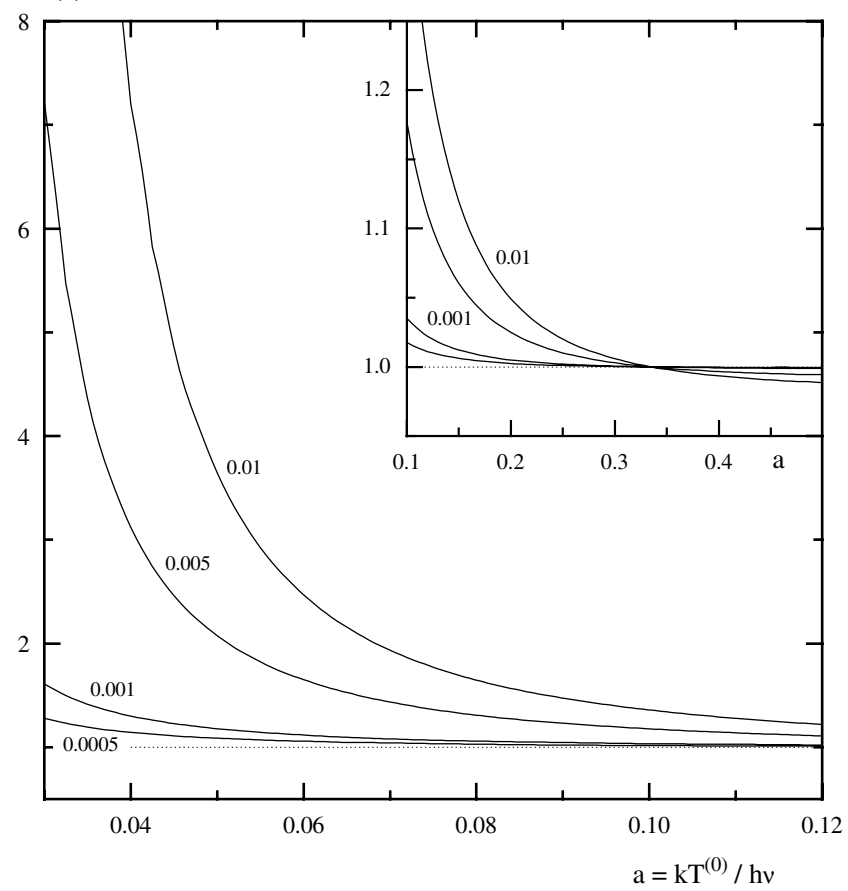

Fig. 1. The values of the distortion function $f_{v}(a, \eta)$ for absorption coefficient (13). The numbers denote the square values of the level of the temperature fluctuations $\eta^{2}$ (see Eq. (6)).

In the Rayleigh-Jeans limit ( $a=k T / h v \gg 1)$ Eq. (9) tends to the expression

$f_{v}\left(\eta^{2}\right) \rightarrow \frac{\left\langle T^{\gamma}\right\rangle}{T^{(0)}\left\langle T^{(\gamma-1)}\right\rangle} \cong \frac{1+0.5 \eta^{2} \gamma(\gamma-1)}{1+0.5 \eta^{2}(\gamma-1)(\gamma-2)}$.

The exact numerical calculations of the function $f_{v}\left(T^{(0)}, \eta\right)$ are presented in Fig. 1 for the $\alpha_{v}$ - coefficient (13) at $\eta^{2}=$ $0.0005,0.001,0.005$, and 0.01 . The results for the case of the absorption coefficient (10) are given in Figs. 2 (for $\eta^{2}=0.001$ ) and 3 (for $\eta^{2}=0.005$ ). They present the $f_{v}-$ coefficient up to $a=$ 0.5 , i.e. include the maximum of Planck's intensity at $a=0.2$. In Fig. 4 we present the $f_{v}(a, \eta)$ - values for the absorption coefficients of the type $\alpha_{v}(T)=D_{v} T^{\gamma}$ with $\gamma=-7,-5,-3,3,5,7$ at $\eta=0.1$. The distortion of Plank's spectra by the temperature fluctuations (see Eq. (8)) with the level $\eta=0.1$ is presented in Fig. 5.

For convenience, we present the distortion coefficients $f_{v}$ for values $a=0.06,0.1,0.2$ and 50 in Tables $1-3$. Remember that $a=0.2$ corresponds to the maximum of the Planck function and $a=50$ practically corresponds to the Rayleigh-Jeans limit. These tables allow us to directly estimate the coefficients $f_{v}$ for various values of the parameters $a, b$, and $\eta$.

The results of calculations demonstrate that the distortion coefficient $f_{v}>1$ in the far ultraviolet region $(a \leq 0.06)$, independent of the form of the absorption coefficient $\alpha_{v}(T)$. This means that the temperature fluctuations give rise to the excess of the ultraviolet radiation.

At the maximum of Planck's function $(a=0.2)$, the distortion coefficient $f_{v}$ can acquire both $f_{v}>1$ and $f_{v}<1$ values depending on the particular form of $\alpha_{v}(T)$. For coefficients (10) and (13), we have $f_{v}>1$. For $\alpha_{v} \propto T^{\gamma}$ we have the coefficient $f_{v}>1$ at $\gamma \geq 0$ and $f_{v}<1$ at $\gamma<0$. At the Rayleigh-Jeans limit $(a \gg 1)$ the $\alpha$ - coefficient (10) gives rise to $f_{v}>1$, and the coefficient (13) gives $f_{v}<1$. For the absorption coefficient $\alpha_{v} \propto T^{\gamma}$, we have $f_{v}<1$ at $\gamma \leq 0$ and $f_{v}>1$ at $\gamma>0$.

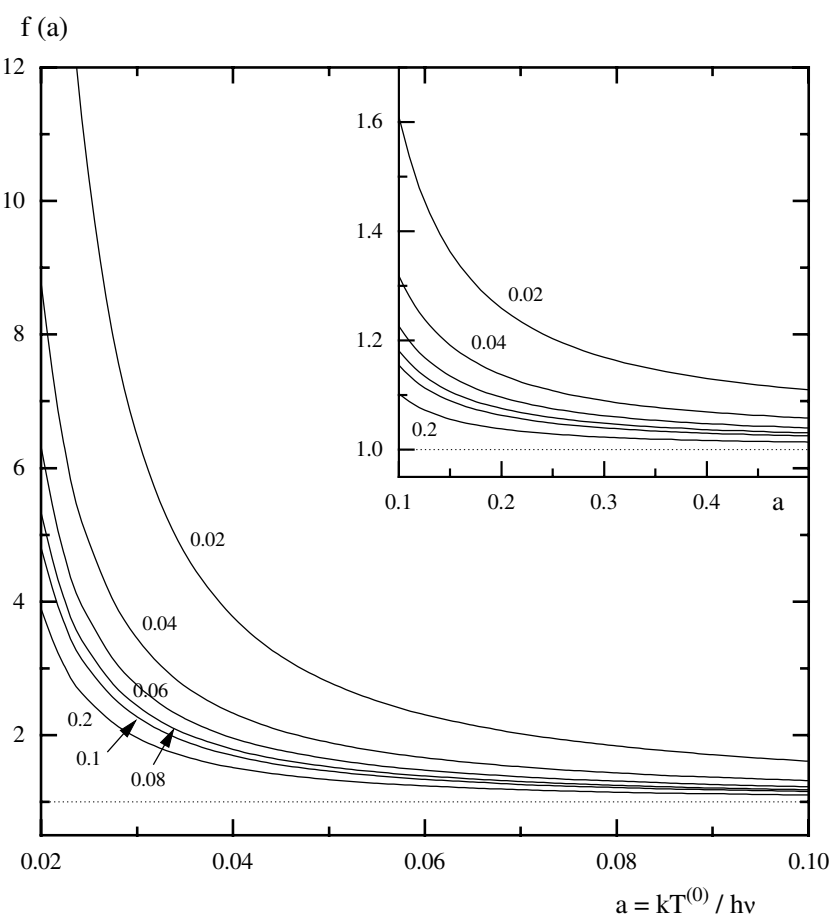

Fig. 2. The values of the distortion function $f_{v}(a, \eta)$ for absorption coefficient (10). The square of the level of the temperature fluctuations $\eta^{2}=0.001$. The numbers denote the values of parameter $b$ (see Eq. (12)).

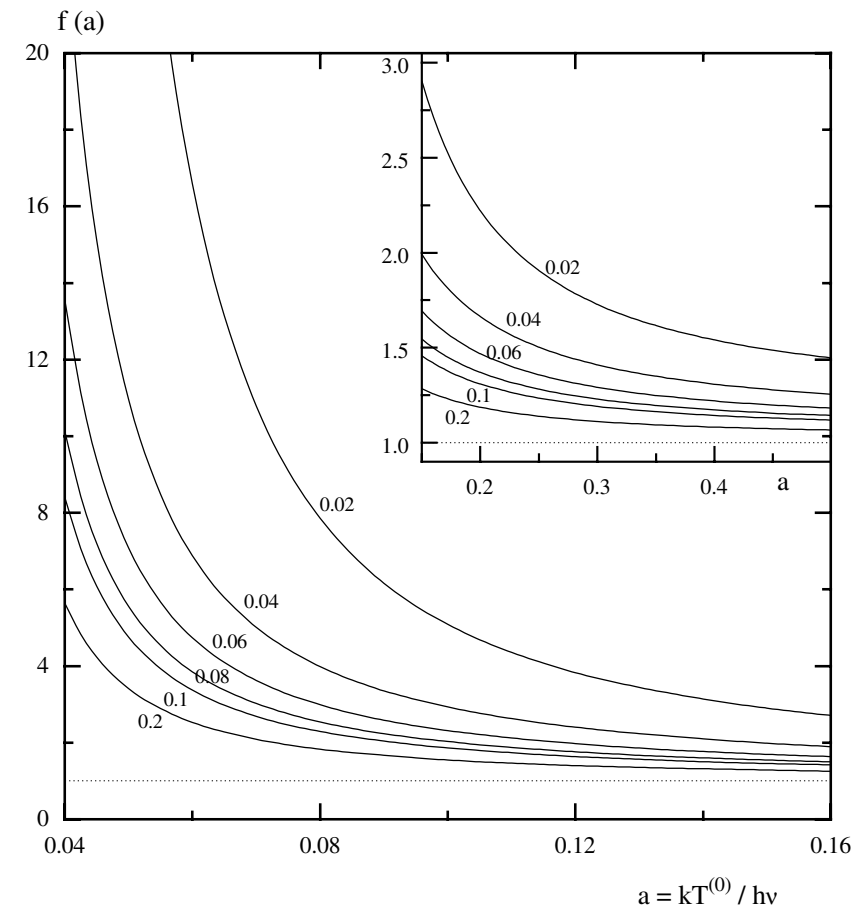

Fig. 3. Same as in Fig. 2, but for $\eta^{2}=0.005$.

Numerically these results strongly depend on the degree of temperature fluctuations $\eta$. The increase in $\eta$ gives rise to more profound deviations from the Planck function $B_{v}\left(T^{(0)}\right)$.

\section{Discussion and summary}

The stochastic temperature fluctuations mutually exist in various media - the stellar photospheres and atmospheres, in the 


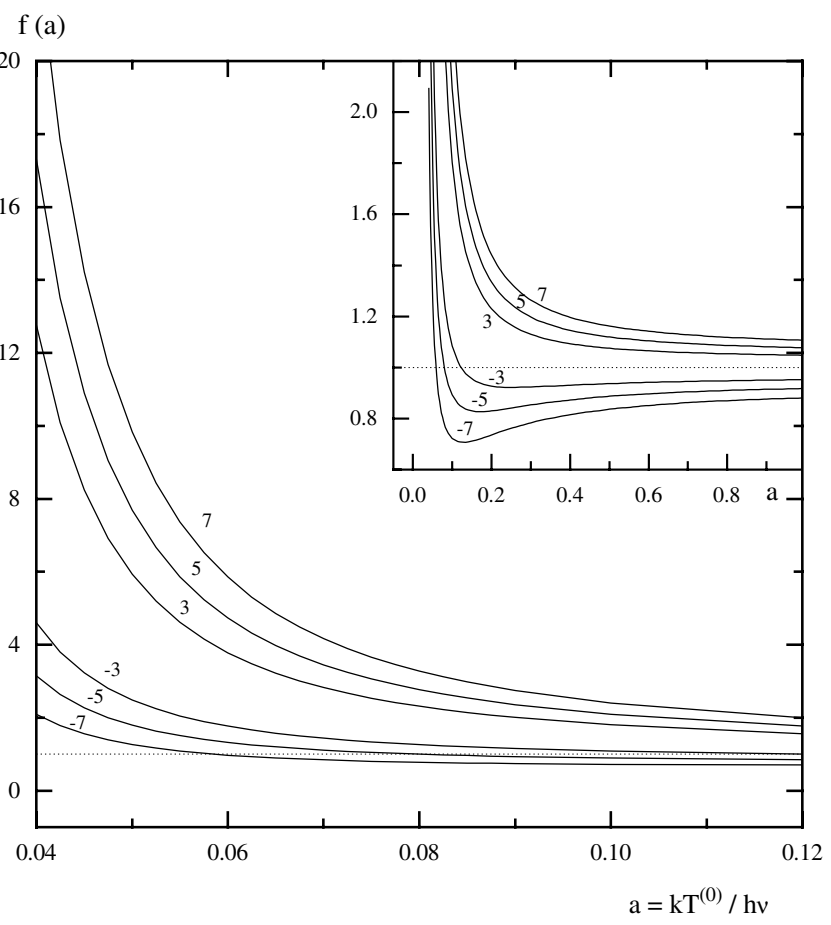

Fig. 4. The distortion function $f_{v}(a, \eta)$ for absorption coefficient $\alpha_{v}(T)=$ $D_{v} T^{\gamma}$. The level of the temperature fluctuations $\eta=0.1$. The numbers denote the values of $\gamma$.

interstellar medium, in AGN, and quasars. In many cases we observe a large number of turbulent (in general, stochastic) "cells". This means that we observe the mean intensity $I_{v}^{(0)}$. The mean intensity does not coincide with the intensity corresponding to the mean temperature $T^{(0)}$. The intensity $I_{v}^{(0)}$ depends on the mean temperature $T^{(0)}$ and the level of temperature fluctuations $\eta=\sqrt{\left\langle\left(T^{\prime}\right)^{2}\right\rangle} / T^{(0)}$. The value $\eta$ is low in the Sun's photosphere $(\eta \simeq 0.03$ ). We do not know how much $\eta$ is in various media mentioned above. It seems $\eta$ is greatest in various situations when energy escape exists more freely from the turbulent cells than in Sun's photosphere.

It is necessary to investigate the influence of the temperature fluctuations in the form of observed spectra. In turbulent (more generally, stochastic) media, the mean intensity $I_{v}^{(0)}$ satisfies the usual radiative transfer equation with the effective (renormalized) kinetic coefficients; in particular, the usual absorption coefficient $\alpha_{v}(T)$ has to be replaced by the effective absorption coefficient that depends on $\eta$. The usual source function - Planck's intensity $B_{v}(T)$ also has to be replaced by the effective source in the form $f_{v}\left(T^{(0)}, \eta\right) B_{v}\left(T^{(0)}\right)$. The coefficient $f_{v}$ describes the distortion of Planck's function due to the existence of the temperature fluctuations.

We presented this investigation for the various types of the absorption coefficients $\alpha_{v}(T)$ at different values of $\eta \leq$ 0.1 . It was found (see Tables 1-3) that the distortion coefficient $f_{v}$ positive and large (can be $\gg 1$ ) in the ultraviolet range of wavelengths. It grows with the decrease in the parameter $a=k T^{(0)} / h v$. Because the Planck intensity at $a \ll 1$ is a very steep function of the temperature, even very small temperature fluctuations give rise to the large increase in the mean intensity. The value $a$ when the coefficient $f_{v}$ begins to grow drastically depends on the particular form of the absorption coefficient, so the value $f_{v} \approx 2$ occurs at $a \simeq 0.09$ for $\alpha_{v} \propto T^{3}$ and at $a \simeq 0.05$ for $\alpha_{v} \propto T^{-3}$.

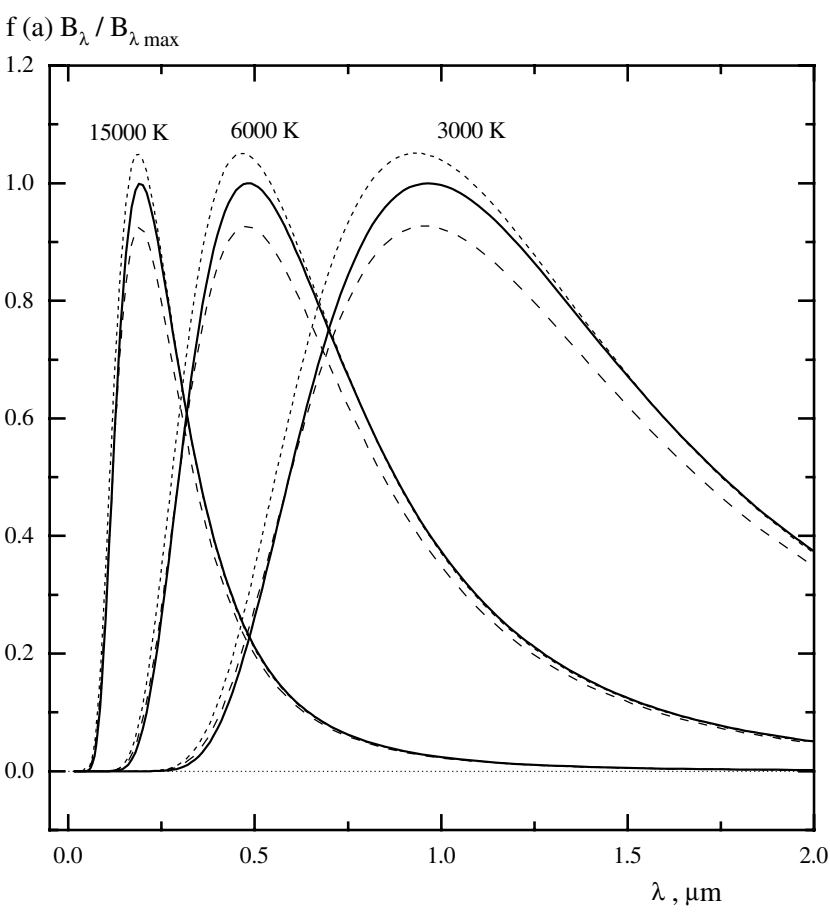

Fig. 5. The distortion of Plank's spectra by the temperature fluctuations (see Eq. (8)) with the level $\eta=0.1$. The solid lines present the Planck spectra $B_{v}\left(T^{(0)}\right)$. The upper dotted lines present the distorted spectra for absorption coefficient (13). The low short - dashed lines present the distorted spectra for absorption coefficient $\alpha_{v}(T) \propto T^{-3}$. The numbers denote the mean temperature $T^{(0)}$.

Table 1. The distortion coefficient $f_{v}$ for the case of the absorption coefficient (13).

\begin{tabular}{llllll}
\hline \hline$a$ & $\eta=0.022$ & 0.032 & 0.071 & 0.1 & 0.224 \\
\hline 0.06 & 1.058 & 1.117 & 1.651 & 2.468 & 15.417 \\
0.10 & 1.018 & 1.035 & 1.177 & 1.357 & 2.900 \\
0.20 & 1.002 & 1.005 & 1.025 & 1.049 & 1.223 \\
50 & 0.999 & 0.998 & 0.992 & 0.985 & 0.909 \\
\hline
\end{tabular}

Table 2. The distortion function $f_{v}$ for the absorption coefficient (10).

\begin{tabular}{llllllll}
\hline \hline$\eta$ & $a$ & $b=0.02$ & 0.04 & 0.06 & 0.08 & 0.1 & 0.2 \\
\hline 0.0316 & 0.06 & 2.304 & 1.660 & 1.474 & 1.387 & 1.336 & 1.238 \\
& 0.1 & 1.610 & 1.318 & 1.226 & 1.181 & 1.155 & 1.102 \\
& 0.2 & 1.259 & 1.137 & 1.096 & 1.075 & 1.063 & 1.038 \\
& 50 & 1.046 & 1.024 & 1.016 & 1.012 & 1.010 & 1.005 \\
\hline 0.0707 & 0.06 & 16.601 & 6.881 & 4.740 & 3.851 & 3.373 & 2.532 \\
& 0.1 & 5.086 & 2.929 & 2.314 & 2.028 & 1.736 & 1.550 \\
& 0.2 & 2.221 & 1.664 & 1.469 & 1.370 & 1.310 & 1.188 \\
& 50 & 1.183 & 1.105 & 1.074 & 1.057 & 1.047 & 1.024 \\
\hline
\end{tabular}

In the maximum of the Planck spectrum and further, in the infrared region of the wavelengths, the function $B_{v}(T)$ is not steep and the distortion of the spectra is mostly due to the particular temperature dependence of the absorption coefficient. The steeper this dependence, the larger distortion due to the temperature fluctuations. If the absorption coefficient grows with the increase in the temperature, then the mean intensity $I_{v}^{(0)}$ is higher than $B_{v}\left(T^{(0)}\right)$. If the $\alpha_{v}(T)$ - coefficient decreases with the growth of the temperature, then the deviation is opposite $\left(f_{v}<1\right)$. For a rather large level of the temperature fluctuations $(\eta \leq 0.1)$, the difference between the mean intensity and $B_{v}\left(T^{(0)}\right)$ 
Table 3. The distortion coefficient $f_{v}$ for the absorption coefficient of the form $\alpha_{v} \propto T^{\gamma}$ at $\eta=0.1$.

\begin{tabular}{llllllll}
\hline \hline$a$ & $\gamma=-7$ & -5 & -3 & 0 & 3 & 5 & 7 \\
\hline 0.06 & 0.973 & 1.326 & 1.768 & 2.454 & 3.780 & 4.733 & 5.856 \\
0.10 & 0.722 & 0.892 & 1.084 & 1.385 & 1.803 & 2.092 & 2.407 \\
0.20 & 0.736 & 0.830 & 0.927 & 1.068 & 1.230 & 1.335 & 1.441 \\
50 & 0.922 & 0.946 & 0.998 & 1.000 & 1.030 & 1.049 & 1.067 \\
\hline
\end{tabular}

at the maximum of the spectrum can reach $\approx 5-30 \%$, both positive and negative.

The calculated values of the distortion coefficient $f_{v}$ and its approximate analytical expression (21) can be used to estimate of the additional terms in the colour indices $U-B, B-V$ etc. For example,

$(U-B)_{\mathrm{add}}=-2.5 \log \frac{f_{v}(\lambda=0.34 \mu \mathrm{m})}{f_{v}(\lambda=0.44 \mu \mathrm{m})}$.

If the observed values of the colour indices differ from the Planck values, then one can explain the difference by the influence of the temperature fluctuations and estimate the needed level of the fluctuations. Of course, such an explanation is not unique, and other explanations may exist.

The large distortion of the continuum spectra in the region $k T / h v \leq 1$, due to the temperature fluctuations, can be included in the usual hydrostatic photosphere theory as a possible explanation of observed ultraviolet excesses. As a result, in this theory the new physical function $\eta$ arises - the level of the temperature fluctuations.

It seems that in modern hydrodynamical models (see Freytag \& Salaris 1999; Asplund et al. 2000, 2004; Nordlund \& Stein 2001; Freytag et al. 2002; Steffen \& Holweger 2002 etc.) of stellar atmospheres, the effects of the temperature fluctuations are included ab initio, as the result of numerical solution of the full system of hydrodynamical and radiative transfer equations. Of course, to obtain the mean spectra, the numerical solutions are to be averaged during a large enough time for the calculations (corresponding to many statistical realizations). Note that the hydrodynamical non-stationary theory is very complex and, up to now, their general application has been limited (see Ludwig \& Kucinskas 2004). The most important achievements of this theory are the corrections of the chemical elements distribution inside the atmospheres.

It should be noted that our simple method can be used for any type of radiation source, not only for the thermal Planck radiation. The temperature fluctuations change the spectra for all sources. The simple semi-analytical theory presented here can be used for the estimation of the level of the temperature fluctuations for various cosmic objects.

Acknowledgements. The authors are very grateful to the anonymous referee and Dr. T. Forveille for many useful remarks.

\section{References}

Asplund, M., Ludwig, H.-G., Nordlund, A., \& Stein, R. F. 2000, A\&A, 359, 681 Asplund, M., Grevesse, N., Sauval, A. J., Allende Prieto, C., \& Kiselman, D. 2004, A\&A, 417, 751

Gray, D. F. 1992, The observation and analysis of stellar photospheres (Cambridge: Cambridge University Press), 134

Freytag, B., \& Salaris, M. 1999, ApJ, 513, L49

Freytag, B., Steffen, M., \& Dorch, B. 2002, AN, 323, 213

Levshakov, S. A., \& Kegel, W. H. 1997, MNRAS, 288, 787

Ludwig, M., \& Kucinskas, A. 2004 [arXiv: astro-ph/0409712]

Mihalas, D. 1978, Stellar atmospheres (San Francisco: Freeman \& Co.), 106

Nordlund, A., \& Stein, R. F. 2001, ApJ, 546, 576

Rybicki, G. B., \& Lightman, A. P. 1979, Radiative processes in astrophysics (N.Y.: Wiley \& Sons)

Silant'ev, N. A. 2005, A\&A, 433, 1117

Silant'ev, N. A., Lekht, E. E., Mendoza-Torres, J. E., \& Rudnitskij, G. M. 2006, A\&A, 453, 989

Sobolev, V. V. 1969, Course in Theoretical Astrophysics, National Aeronautic and Space Administration, NASA technical translation, NASA TT F-531

Steffen, M., \& Holweger, H. 2002, A\&A, 387, 258

Stix, M. 1991, The Sun, An Introduction (Berlin: Springer Verlag), 207

Van Kampen, N. G. 1981, Stochastic processes in physics and chemistry (North Holland: Amsterdam)

Tatarskii, V. I. 1967, Wave propagation in turbulent atmospheres (in russian) (Moscow: Nauka) 